\title{
LEGITIMACIÓN DE LAS ERT EN CIUDAD DE MÉXICO: UN ANÁLISIS CON EL ENFOQUE DE LA ACCIÓN PÚBLICA
}

\section{LEGITIMATION OF ERT'S IN MEXICO CITY: AN ANALYSIS WITH THE APPROACH OF PUBLIC ACTION}

\author{
Yasmín Hernández Romero*
}

RESUMEN

El objetivo de este artículo consiste en analizar la regulación de las empresas de redes de transporte basadas en aplicaciones móviles (ERT) en la Ciudad de México (CDMX), mediante el enfoque de la acción pública propuesto por Lascoumes y Le Galés (2014), basándose en documentos oficiales e hipertextos, reconstruyendo el proceso acontecido durante el periodo de 2013 a 2017, el cual permitió su operación legal. Se concluye explicando en cómo la configuración de la acción pública y el arreglo final establecido condujeron a la legitimación y legalización de las ERT.

PALABRAS CLAVE: MÉXICO * LEGISLACIÓN * TRANSPORTE URBANO * GRUPOS DE INTERESES * TELÉFONO MÓVIL

\section{ABSTRACT}

The aim of this article is to analyze the regulation of the mobile application based transport network companies (ERT) in Mexico City (CDMX), through the public action approach proposed by Lascoumes and Le Galés (2014), based on official documents and hypertexts, reconstructing the process occurred during the period from 2013 to 2017, which gave way to legal operation. Concluding on how the configuration of public action and the final settlement established led to the legitimation and legalization of ERT's.

KEYWORDS: MEXICO * LEGISLATION * URBAN TRANSPORT * INTERESTS GROUPS * MOBILE TELEPHONE 


\section{INTRODUCCIÓN}

Actualmente, la incursión de las Empresas de Redes de Transporte basadas en aplicaciones móviles (ERT) como Uber y Cabify, en México y en el mundo, ha dado lugar a la emergencia de un nuevo paradigma en la prestación del servicio de transporte de pasajeros en la modalidad individual. El reconocimiento otorgado a las eRT en Ciudad de México (сDмx) constituye un tema de relevancia pública, al ser la primera entidad de México y de América Latina en la que se logra la regulación de este servicio.

El tema de las ert en México ha estado presente de forma continua en periódicos nacionales y redes sociales; sin embargo, en el ámbito académico es escasa su discusión y análisis. Las publicaciones encontradas han seguido diversos ejes de discusión. Desde un enfoque económico, se delibera sobre la pertenencia del servicio de Uber $y$ del taxi tradicional en el mercado relevante (Ávalos y Vásquez, 2015; González de Cossío, s.f.); se indaga la percepción de los usuarios sobre el transporte bajo demanda en una de las entidades federativas del país (Moreno et ál, 2016); se establecen las relaciones de trabajo en el modelo de gestión del servicio que ofrece Uber (Hernández y Galindo, 2016). Desde un enfoque jurídico, se expone la discusión legal en los Estados que han mostrado mayor resistencia a la penetración de las ERT, como lo es Yucatán ${ }^{1}$ (Parra, 2017). También, se abordan las barreras sociales $y$ legales en distintas entidades de México (Law@Tec, 2016).

En este contexto, se busca analizar el proceso a partir del cual las ERT lograron establecerse como un servicio legitimado, previo a legalizarse en la сDмx. No obstante, para poder explicar la regulación de las eRT en la CDMx, es necesario presentar algunos señalamientos en torno al servicio público de transporte en la modalidad individual o sin ruta fija, conocido

1 En la ciudad de Mérida se ha exigido a Uber que se registre como un sistema de transporte, empero la empresa ha presentado un amparo para evitar esta resolución, por lo que técnicamente se trata de un servicio irregular. Cabify sí acató tal disposición, convirtiéndose en la primera plataforma en obtener su registro ante el gobierno estatal. como servicio de taxi ${ }^{2}$; principal competidor de las ERT. Se entiende por taxi a:

... el vehículo automotor de alquiler con conductor, sin itinerario fijo, destinado al transporte de uno o varios pasajeros - por lo general no más de cuatro- dentro del esquema de servicio público y, en consecuencia, con sujeción a un régimen de derecho público que impone los requisitos que debe cubrir el vehículo y su conductor, sus reglas de operación, así como la tarifa que debe aplicarse (Fernández citado en Abascal, 2009, pp. 229-230).

Históricamente, la prestación del servicio de transporte de personas ha sido considerada una facultad de la autoridad gubernamental, al atender una necesidad pública. De acuerdo con Medina (2013), en México, desde sus orígenes, la prestación del servicio de coches de alquiler estuvo mediada por el permiso del Gobierno Virreinal. Más tarde, serían los gobiernos de cada entidad federativa los encargados de la regulación de este servicio tratándose de vías primarias y secundarias locales, sin injerencia en vías federales.

La Constitución Política de los Estados Unidos Mexicanos establece, en el artículo 28, que el Estado podrá concesionar ${ }^{3}$ la prestación de servicios públicos, conforme a las normas del derecho público general $y$ de cada entidad federativa en particular. En este caso, la autoridad gubernamental de cada entidad tendrá el derecho y obligación de regular y controlar la prestación

2 En cDmx, la Ley de Movilidad del Distrito Federal establece que el servicio de transporte se clasifica en: de carga y de pasajeros. Este último se divide en público, mercantil, privado y particular. A su vez, el servicio público se subdivide en masivo, colectivo, individual (dentro del cual se ubica el taxi) y ciclotaxis.

3 El Gobierno Estatal tiene la facultad de prestar el servicio por sí o a través de concesiones y permisos que otorgue a personas físicas o morales. La concesión es el acto administrativo mediante el cual la autoridad en materia de transporte confiere el derecho y las obligaciones correlativas de la prestación del servicio público. 
de dicho servicio, en lo que se refiere a número de taxis, territorios de operación, bases, tarifas y modelos de autos. Asimismo, en el artículo 115 de esta Constitución se dispone que:

... los Estados adoptarán, para su régimen interior, la forma de gobierno republicano, representativo, popular, teniendo como base de su división territorial y de su organización política y administrativa, el Municipio Libre... Los municipios, en los términos de las leyes federales y Estatales relativas estarán facultados para:... h) Intervenir en la formulación y aplicación de programas de transporte público de pasajeros cuando aquellos afecten su ámbito territorial.

Como una nueva modalidad de servicio, en el año 2013, las ert como Uber y Cabify, incursionan en la cDmx. Son empresas que, haciendo uso de una aplicación móvil, enlazan servicios de transporte individual de pasajeros ofertados por particulares no concesionados con usuarios que lo soliciten. Dichas empresas, por medio de la misma aplicación, manejan acceso a facturación y pago con tarjeta de crédito por el servicio prestado.

En la cDmx, como en casi todas las ciudades en las que han incursionado las ERT, su presencia ha suscitado un debate en torno a la legalidad de esta modalidad de servicio de transporte. Las posturas en discusión giran en torno a: 1) quienes afirman que se trata de modalidades de transporte equiparables al taxi, en tanto que ambos prestan servicio de transporte de pasajeros y 2) quienes sostienen que son modalidades de transporte de naturaleza distinta al taxi, con nichos de mercado diferenciados.

Las diversas posturas dieron lugar a bloqueos y manifestaciones por parte de los operadores de taxi, destacando en los medios de comunicación, los eventos acontecidos en octubre de 2014 y en mayo de 2015. Por su parte, los representantes de las ERT hicieron uso de las redes sociales para la promoción y descrédito del servicio de taxi tradicional. Los actos de violencia $y$ vandalismo entre taxistas $y$ operadores de las ERT no se hicieron esperar. Las disputas entre los prestadores de ambos servicios y la presión de la opinión pública, encontraron un espacio de expresión en el foro de discusión realizado los días 17 y 18 de junio de 2015, organizado por el Laboratorio para la Ciudad. Finalmente, el 15 de julio del 2015 se establece un arreglo normativo para regular el servicio de transporte de las ERT en cDmx. Esto se objetiva en el Acuerdo por el que se crea el Registro de Personas Morales que Operen y/o Administren Aplicaciones y Plataformas Informáticas para el Control, Programación y/o Geolocalización en Dispositivos Fijos o Móviles, a través de las cuales los Particulares pueden Contratar el Servicio Privado de Transporte con Chofer en el Distrito Federal. A través de dicho Acuerdo, las ERT podrán coexistir con el servicio de transporte público individual y cada modalidad contará con una reglamentación distinta. Ese primer resultado es un punto de llegada, dentro un amplio proceso, en la construcción del nuevo orden legal para la prestación del servicio de transporte urbano.

Para el desarrollo de este artículo, se ha organizado el análisis en tres apartados. En el primero, se presentan consideraciones generales acerca de la configuración de la cDmx, que sirven de contexto para entender la dinámica $y$ necesidad de transporte que presenta. Aquí se hace énfasis en el servicio de transporte público de pasajeros en la modalidad individual, conocida como taxi, principal competencia del servicio que prestan las ERT.

En el segundo, se expone el enfoque de la acción pública de Lascoumes y Le Galés (2014). A diferencia de los enfoques estatistas, que conciben a las decisiones púbicas de manera vertical y como una cuestión de orden técnico, el enfoque de la acción pública de los autores en mención, da cuenta de esta como un proceso colectivo, cuyos arreglos entre los actores públicos y privados intervinientes, en un marco de instituciones, hacen de la decisión un asunto político, en entornos caracterizados por la incertidumbre.

En el tercer apartado, bajo el marco de análisis de Lascoumes y Le Galés (2014), se refiere la dinámica generada desde la incursión de las eRT en la cDmx hasta su regulación. Como estrategia de abordaje del tema en cuestión se 
trabajó con documentos oficiales e hipertextos, a partir de los cuales se reconstruye el proceso que dio paso al establecimiento de un orden que legaliza el funcionamiento de las ert en cDMx. Al final, se presentan algunas reflexiones a manera de conclusión.

\section{CONFIGURACIÓN DE LA CIUDAD DE MÉXICO}

La cDmx es una de las 32 entidades federativas en que se divide políticamente los Estados Unidos Mexicanos, es también sede de los Poderes de la Unión y capital del país. Dicha entidad constituye el principal centro político, económico, académico y cultural de México. Debido a su relevancia, se ha caracterizado por ser pionera en distintas políticas y programas, así como ha marcado la pauta para otras entidades.

Esta entidad posee un carácter sui generis en su administración y gobierno, por lo que es pertinente incluir una breve reseña de su conformación. Desde la creación del Distrito Federal, en 1824, se acordó su establecimiento en cDmx, siendo asiento de los poderes federales. En el año 1928, se crea el Departamento del Distrito Federal, con carácter administrativo. El Jefe de este Departamento, conocido como Regente, durante mucho tiempo fue nombrado por el Presidente de la República.

Esta situación se modifica en 1996, cuando se crea el Gobierno del Distrito Federal. A partir de este momento, el Jefe de Gobierno es elegido mediante votación ciudadana, con una duración de seis años en el cargo. Desde entonces han coexistiendo en esta ciudad dos órganos de gobierno, el federal y el del Distrito Federal (ahora Ciudad de México ${ }^{4}$ ), procedentes de partidos políticos distintos.

4 En el año 2016, el Distrito Federal pasó a ser entidad federativa $y$ se cambia el nombre de esta entidad por el de CDMx. Políticamente, se divide en 16 delegaciones (designadas en la nueva Constitución de la CDMX como alcaldías a partir de 2018). De acuerdo con el marco legal de la CDMX, las autoridades locales están conformadas por la Asamblea Legislativa (Congreso de la Ciudad de México a partir de septiembre de 2018),
El primer Jefe de Gobierno del Distrito Federal es elegido en 1997. Desde esa fecha, el titular del Gobierno del Distrito Federal ha provenido del Partido de la Revolución Democrática (PRD), partido político nacional de izquierda. De ese periodo a la fecha, a nivel federal, el gobierno ha recaído en dos partidos políticos. El Partido Revolucionario Institucional (PRI) quien gobernó durante el periodo 1996-2000 y el Partido Acción Nacional (paN), en los dos sexenios siguientes, de 2000 a 2006 y de 2006 a 2012; en el periodo actual, 2012-2018, el gobierno federal es nuevamente de extracción priista.

En el aspecto estadístico, de acuerdo con información del año 2015, proveniente del Instituto Nacional de Estadística y Geografía (INEGI), la cDmx posee una superficie territorial de $1495 \mathrm{~km}^{2}$; con 8918653 habitantes, que representan el 7,5\% del total del país; de los cuales 4687003 son mujeres y 4231650 son hombres ocupando el segundo lugar a nivel nacional por el número de habitantes. En términos de densidad de población, ocupa el primer lugar en el país, con 5967 personas por $\mathrm{km}^{2}$. En ese mismo año, el $99,5 \%$ de la población de la cDmx se reporta como urbana y solo el 0,5\% como rural. El promedio de escolaridad de la población de esta entidad es del segundo año de educación media superior.

El fuerte dinamismo económico de la cDmx se refleja en la aportación que hace al Producto Interno Bruto (PIB) nacional, ocupando el primer lugar con respecto a las demás entidades federativas. En ese contexto, el transporte constituye una necesidad colectiva $y$ un elemento central en el funcionamiento de esta ciudad, ya que además resulta ser un centro de atracción y de expulsión de población. Como referente de los desplazamientos, se presentan los siguientes datos cuya base es la Encuesta Intercensal realizada en 2015 por el INEGI.

el Jefe de Gobierno de la Ciudad de México y el Tribunal Superior de Justicia. 
TABLA 1

FLUJOS DE POBLACIÓN EN CIUDAD DE MÉXICO

2015

\begin{tabular}{|c|c|c|c|c|c|c|c|}
\hline \multicolumn{4}{|c|}{ POBLACIÓN QUE INGRESA A LA CDMX } & \multicolumn{4}{|c|}{ POBLACIÓN QUE SALE DE LA CDMX } \\
\hline \multirow{2}{*}{$\begin{array}{l}\text { ENTIDAD DE } \\
\text { ORIGEN }\end{array}$} & \multicolumn{3}{|c|}{ MOTIVO DE MOVILIDAD } & \multirow{2}{*}{$\begin{array}{l}\text { ENTIDAD DE } \\
\text { ORIGEN }\end{array}$} & \multicolumn{3}{|c|}{ MOTIVO DE MOVILIDAD } \\
\hline & TRABAJAR & ESTUDIAR & TOTAL & & TRABAJAR & ESTUDIAR & TOTAL \\
\hline Estado de México & 1320748 & 355896 & 1676644 & $\begin{array}{l}\text { Ciudad de } \\
\text { México }\end{array}$ & 170901 & 56756 & 227657 \\
\hline Hidalgo & 24103 & 4491 & 28594 & & & & \\
\hline & 11452 & 3455 & 14907 & & & & \\
\hline Puebla & 12952 & 1483 & 14435 & & & & \\
\hline TOTAL & 1369255 & 365325 & 1734580 & & & & \\
\hline
\end{tabular}

Fuente: Información tomada del Estudio Origen-Destino de la Zona Metropolitana del Valle de México, 2017 (INEGI-UNAM, 2017). Diapositiva 6.

Referente a la clasificación del servicio de transporte de pasajeros en cDmx, la Ley de Movilidad (2014) establece que este servicio se clasifica en: público, mercantil, privado y particular. A su vez, divide al transporte público en masivo, colectivo, individual y ciclotaxis. La infraestructura de transporte público se encuentra conformada por transporte gubernamental $y$ transporte concesionado.

Respecto al movimiento de personas en transporte público, de acuerdo con información de la última Encuesta Origen-Destino en Hogares de la Zona Metropolitana del Valle de México (zмvм) (ЕоD), aplicada por el INEgi en el año 2017, en esta zona conformada por 59 municipios del Estado de México y un municipio de Hidalgo, junto con las 16 delegaciones de la срмx, de los 23,41 millones de viajes que se realizan un día entre semana, 15,57 millones de viajes son en transporte público. De estos, según principales medios de transporte utilizado, $74,1 \%$ se hace en microbús o combi, $28,7 \%$ en metro y $10,5 \%$ en taxi de sitio, calle o aplicación. En la cDmx, de los 12,8 millones de viajes que se realizan un día entre semana, 8,62 millones de viajes son en transporte público de los cuales, según principales medios de transporte utilizado, $67,8 \%$ se hace en microbús o combi, $38,2 \%$ en metro y $11,2 \%$ en taxi de sitio, calle o aplicación.

En la cDmx, de todas las modalidades de transporte público, el taxi es el que cuenta con la mayor cantidad de vehículos registrados de servicio público y concentra el mayor número de concesionarios individuales, debido al esquema de otorgamiento de concesiones ${ }^{5}$ a personas físicas. A diciembre de 2016, se tenían registradas 132538 concesiones, de acuerdo con información de la Dirección General del Servicio de Transporte Público Individual (INFomEx, 2017).

5 Para una explicación más amplia sobre el otorgamiento de una concesión se sugiere revisar el documento realizado por Flor Jiménez Bautista (2009), titulado: Concesión del transporte público individual, así como, los capítulos II Ter y II Cuarter, y capítulos III, IV, v, VI, vII de la Ley de Transporte y Vialidad del Distrito Federal. Y los capítulos IV al viI de la Ley de Movilidad, (2014). 
Normativamente, el servicio de taxi está catalogado como un servicio público. Ello implica que debe regirse por el derecho administrativo de orden público, organizarse y dirigirse por el Estado, pudiendo proporcionarse directamente por este o indirectamente por personas físicas o morales, previo otorgamiento de una concesión. Con base en la Constitución de la сDмx "corresponde al gobierno de la Ciudad autorizar y regular la prestación de servicios públicos de transporte de personas por particulares y las tarifas correspondientes, en los términos que establezca la Ley" (2017, p. 37).

El servicio público individual de pasajeros concesionado (taxi) se divide en dos modalidades, de acuerdo con el Reglamento de Transporte del Distrito Federal (2003): taxi libre $y$ taxi de sitio.

Taxi Libre: vehículo que presta servicio de transporte público individual de pasajeros, sin itinerario fijo, ni adscripción permanente a alguna base de servicio en el ámbito territorial del Distrito Federal. Estos pueden organizarse gremialmente para proporcionar el servicio en bases previamente autorizadas por la Secretaría.

Taxi de Sitio: vehículo que presta el servicio público de transporte individual de pasajeros sin itinerario fijo, a través de espacios físicos autorizados en bases, centros de transferencia modal, terminales $y$ demás lugares que determine la Secretaría (Gobierno del Distrito Federal, 2003, art. 43).

Adicionalmente a los concesionarios y permisionarios mencionados, existen cerca de 30000 automóviles que prestan el servicio con la cromática oficial, pero en la ilegalidad, esto es, sin contar con la concesión respectiva y son conocidos coloquialmente como "taxis piratas". A lo anterior, la incursión de las ERT ha venido a adicionar una amplia flotilla de vehículos en operación para el transporte de pasajeros. Es así, que entre los conductores de las ERT y los concesionarios del servicio de transporte público individual de pasajeros se ha desencadenado una franca confrontación, que se analizará en este artículo con el enfoque de la acción pública.

\section{EL ENFOQUE DE ACCIÓN PÚBLICA}

Jean-Claude Thoening (1997), sociólogo nacido en Suiza, plantea una diferenciación entre el enfoque de política pública y el de acción pública, que se toma como punto de partida en este documento. En el enfoque de política pública subyace un postulado estadocentralista junto a una concepción convencional de lo político, en la que la autoridad es considerada el único o principal actor en la definición de las políticas públicas, cuyo proceso, que va de la definición a la implementación, se considera una secuencia lógica de eventos ${ }^{6}$.

Para Thoening, el estudio de la política pública desde la sociología de la acción posibilita la renovación de la teoría de lo político; en esta línea se ubica la propuesta de Lascoumes (1994, citado en Thoening, 1997). El enfoque de la acción pública asume un postulado distinto, en el sentido de que los actores públicos no tienen la hegemonía ni en lo político, ni en el tratamiento o gestión de los asuntos públicos. Este enfoque es multiactorial, es decir que, la construcción de los problemas públicos y su solución son de carácter colectivo - participando actores públicos y privados-, lo que supone la negociación, pero también la contradicción y discontinuidad en los arreglos que se den.

Así, el proceso decisorio se construye en situaciones y contextos específicos y en múltiples escenarios de acción. Este enfoque se aleja de las posturas que suponen la autonomía del actor frente a las estructuras e instituciones.

La mayoría de los trabajos contemporáneos sobre la acción pública toman en cuenta a los actores, sus intercambios y el sentido que le dan a éstos, pero también a las instituciones, las normas, los

$6 \quad$ En este artículo no sé profundiza en el debate teórico acerca de las políticas públicas, ello rebasa el propósito del texto. Solo se toman aspectos generales al servir de referente para entender las particularidades del enfoque de la acción pública. 
procedimientos que gobiernan el conjunto de las interacciones, así como las representaciones colectivas (Lascoumes y Le Galés, 2014, pp. 15-16).

Bajo este enfoque de observación, la acción pública es vista como un proceso cuyo resultado no puede anticiparse, al derivar de la interacción entre actores, instituciones y representaciones, cuya relación no es lineal. Con fines analíticos y para poder dar cuenta de las variables intervinientes en la acción pública y sus vínculos, Lascoumes y Le Galés proponen el siguiente modelo:

ESQUEMA 1

CINCO VARIABLES DEL ANÁLISIS CON EL ENFOQUE DE LA ACCIÓN PÚBLICA Y SUS COMBINACIONES

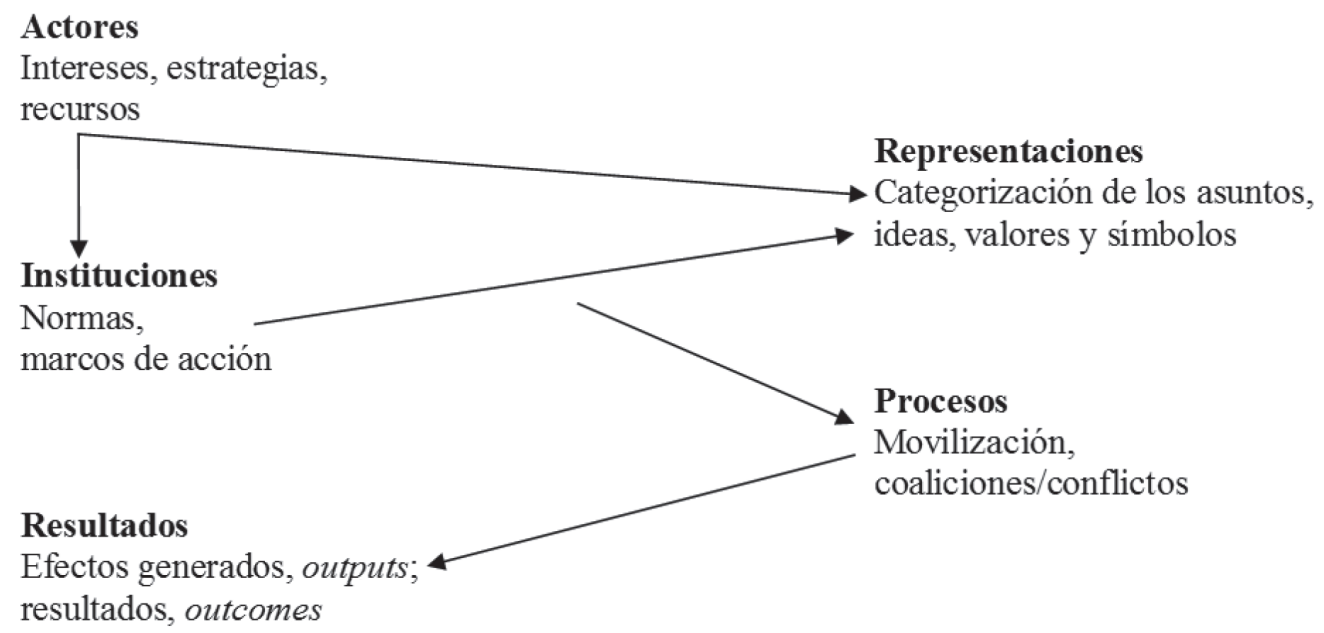

Fuente: Lascoumes y Le Galés, 2014, p. 20.

En una sociedad que se diga democrática, la definición y solución de los problemas públicos se constituyen en un proceso político, en el que intereses y posturas distintas entran en confrontación, por lo que la solución buscará legitimarse. En este punto, siguiendo a Habermas, quien va más allá de los planteamientos weberianos, la legitimidad se funda en consensos construidos a través de un activo diálogo y debate en el espacio público; es decir, que los procedimientos por sí solos no pueden producir legitimación, sino que requieren a su vez, ser legitimados mediante procesos participativos comunicativos (Vergara, 2005), aspecto que se evidencia con claridad en el caso abordado en este artículo. En ese sentido, el enfoque de análisis de la acción pública constituye un recurso heurístico que guía la explicación del proceso de regulación de las ERT en CDMx.

\section{VARIABLES DE ANÁLISIS EN LA REGULACIÓN DE LAS ERT EN CDMX}

En este apartado, se expone la configuración de cada una de las cinco variables que proponen Lascoumes y Le Galés en su esquema de análisis de la acción pública. Para esto, se ha considerado como referente empírico lo acontecido ante la penetración de las ERT en CDMx a partir del año 2013 y hasta el año 2017.

\section{ACTORES}

Dentro de los principales actores intervinientes ante el ingreso de las ERT a la capital de la República, se encuentran los siguientes: 
a) Prestadores del servicio de transporte público individual de pasajeros

La acción pública en torno a la entrada de las ERT no puede entenderse si no se consideran a los prestadores del servicio público de transporte de pasajeros en la modalidad individual, conocida como servicio de taxi ${ }^{7}$. Los concesionarios de transporte público pueden organizarse jurídicamente como asociaciones civiles, sociedades cooperativas o sociedades mercantiles, las cuales tendrán el derecho de representación de sus agremiados ante las autoridades.

Cabe aclarar que, dentro de esta ocupación, no se da una relación de trabajo asalariada basada en contratos laborales, formales o informales, remunerada con sueldos y salarios. Más bien se asemeja a un trabajo independiente o por cuenta propia, al prestar un servicio mediante relaciones mercantiles de compra-venta, cuya remuneración depende de los beneficios del servicio producido; lo anterior, en función de la titularidad o no de los medios para la producción de este.

En la producción del servicio público de transporte no se presenta una relación laboral típica, en la que se preste un trabajo a cambio de un salario. Aquí, las relaciones de trabajo tienen diversos matices, dependiendo de las combinatorias de los elementos de titularidad de la concesión, propiedad del vehículo y operación del servicio (Hernández y Galindo, 2016, p. 167).

Para poder comprender la particular actuación de los también llamados taxistas tradicionales, se incorpora un elemento conceptual adicional: el de la identidad. La identidad es un concepto relacional, en tanto que, se construye en relación con el "otro" o los "otros". A partir de la identidad se genera un

$7 \quad$ En este documento se considera de manera unívoca a los prestadores del servicio tradicional de taxi; sin embargo, en otros trabajos se ha profundizado en la heterogeneidad de intereses que conforman este grupo (Hernández y Galindo, 2013). sentido de pertenencia a un grupo, condición para llevar a cabo una acción organizada.

Siguiendo a Pogliagini y refiriéndose a los taxistas tradicionales, la identidad de este gremio no es siempre la misma, debido a que se establece según los intereses en disputa, pudiendo clasificarse de acuerdo con la jerarquía y el nivel de análisis:

Por lo tanto, el "nosotros" y el "otros" también será diferente. En los taxistas, en el nivel de agregación más amplio el nosotros se conforma de "nosotros los taxistas" en oposición a "el otro gobierno". En un nivel intermedio, "nosotros los taxistas organizados" en distinción a "los otros los taxistas libres". Y en un nivel más concreto "nosotros los taxistas de la organización o base A" en diferenciación de "los otros los taxistas de la organización o base B” (2012, p. 287).

En la cDmx existen alrededor de 150 organizaciones formales de taxistas, que controlan 657 bases de taxis. De manera regular, dichas organizaciones no requieren mantener vínculos, sin embargo, la llegada de las ERT ha sido un detonante para que el gremio de taxistas comience una organización de mayor escala, no solo a nivel de entidad federativa, sino también a nivel nacional y con otros países, toda vez que la penetración de las ERT ha generado conflictos similares en otras ciudades del mundo. Así, a mediados de 2015, Taxistas Organizados de la Ciudad de México (тосрмx) se unieron con la Asociación Élite Taxi de Madrid, el lema de esta $y$ otras organizaciones de taxistas a nivel internacional es "Uber off". A finales de 2016, ante la penetración de las ERT en otras ciudades de México, se integra el Movimiento Nacional del Taxi República Mexicana.

b) Autoridades de gobierno en materia de transporte

En el ámbito de la administración pública, la regulación del servicio público de transporte corresponde a la autoridad estatal. En la cDmx, desde el 30 de abril de 2013, la dependencia encargada del control y administración de este 
servicio es la Secretaría de Movilidad (semovi), antes Secretaria de Transporte y Vialidad (sETRAVI). Dicha Secretaría es la encargada de autorizar y vigilar el número de unidades en circulación, modelo de vehículos, territorios de operación, bases, tarifas y demás aspectos administrativos necesarios para su funcionamiento.

\section{c) Empresas de Redes de Transporte}

Las ERT son empresas tecnológicas que ofrecen una aplicación electrónica de intermediación de transporte bajo demanda; esto quiere decir que su propósito es enlazar servicios de transporte privado mediante el uso de un dispositivo móvil (smartphone) con usuarios potenciales. En el caso de Uber y Cabify, dicho servicio es vendido a terceros, propietarios de vehículos particulares, para proveer el servicio de transporte a usuarios registrados en la aplicación. En México, existen otras erT como Easy Taxi, Taxibeat, Yaxi, Avant, CityDrive, Jetty y Urbvan, estas dos últimas en el transporte privado colectivo, $y$ algunas que han integrado al modelo de taxi tradicional, enlazando a usuarios con taxistas concesionados, también, mediante una aplicación tecnológica.

Uber y Cabify son empresas internacionales, la primera se origina en San Francisco, California; la segunda se funda en España. Ambas empresas cuentan con voceros que las representan en cada país en el que penetran, quienes son los autorizados para dirigirse a los medios de comunicación. Así, es necesario tener en cuenta la distinción de los diversos grupos que al interior de la ERT tienen cabida, los representantes, los socios propietarios $y$ que a la vez son operadores, $y$ los que solo son conductores.

\section{d) Comisión Federal de Competencia} Económica (COFECE)

La COFEce es un órgano autónomo cuya misión es garantizar la competencia y libre concurrencia en el mercado mexicano; así como también el prevenir, investigar y combatir los monopolios. Dentro de sus facultades está el ordenar medidas para eliminar las barreras a la competencia y libre concurrencia.

En el caso particular que se aborda, con fecha 4 de junio de 2015, la cofece emite una opinión dirigida a los Gobernadores de los Estados, el Jefe de Gobierno del Distrito Federal $y$ las Legislaturas de las entidades federativas, respecto al impacto favorable de los servicios de transporte de personas por medio de plataformas móviles en el proceso de libre concurrencia y competencia económica. Dicha opinión fue de gran relevancia en la posterior regulación de las ERT en la CDMx.

\section{e) Usuarios}

En la producción del servicio de transporte de pasajeros, los usuarios son parte fundamental. En el caso del servicio público, el usuario "entra en relación directa con el concesionario y sólo de manera excepcional con el órgano público concedente" (Ramos, 2011, p. 27). Una diferencia entre el servicio público de transporte y el servicio de transporte privado es que en este último, los usuarios evalúan el servicio al terminar el viaje y dicho reporte se envía a la ERT. En este caso, aplica lo señalado por De la Garza (2013), en cuanto a la participación del cliente (usuario) en los trabajos no clásicos, quien es un elemento inmerso en el proceso de producción de servicios al participar en la supervisión del trabajo (prestación del servicio).

Existen otros actores que se van adicionando al proceso de legalización de las ERT, los cuales se irán describiendo en el punto relativo al proceso. De manera solo enunciativa se mencionan en este cierre a: Institutos Políticos, Comisión de Derechos Humanos del Distrito Federal, comunicadores y analistas.

\section{REPRESENTACIONES}

Hablar de representaciones sociales implica dar cuenta de la concepción que tienen los individuos sobre algo, la cual se forma a partir del conocimiento de sentido común. Se trata de universos de opinión que guían y regulan las prácticas sociales en el ámbito de la cotidianidad. Las representaciones son una especie de 
"teoría profana" que sirve para categorizar a las personas y sus comportamientos (Mora, 2002). Llevado al ámbito de la acción pública, las representaciones son "ciertas formas de discursos argumentados que orientan y legitiman las posiciones de los principales actores y los grandes arbitrajes públicos" (Lascoumes y Le Galés, 2014, p. 62).

De manera general, la categorización del transporte público tradicional (taxi) se vincula con las siguientes opiniones: inseguridad, arbitrariedad en el cobro, unidades deterioradas, conductores descorteses, por mencionar solo algunos aspectos, lo cual se resume en una falta de calidad en el servicio. A ello se agrega la imagen de corrupción que gira en torno al otorgamiento de concesiones, así como a la supervisión y aplicación de la normatividad por las autoridades gubernamentales en materia de transporte (Ávalos y Vásquez, 2015; Law@Tec, 2016).

La contraparte del servicio tradicional lo representan las ERT, asociadas con: seguridad, tarifa transparente, unidades de transporte con modelos recientes y conductores afables con el usuario (Ávalos y Vásquez, 2015; Moreno et ál, 2016; Law@Tec, 2016). Por lo menos estos fueron los adjetivos con los cuales el servicio de transporte privado de empresas como Uber y Cabify logró penetrar en la cDmx. Lo anterior, conlleva una idea del buen manejo por parte de la empresa privada versus la lógica que priva en empresas donde el gobierno tiene participación. En términos generales, se puede decir que el servicio de las ERT se vincula con la modernización en el sector transporte, mientras que el servicio público representa lo anquilosado.

Además de la representación que tienen los "otros", también está aquella que los individuos o grupo se hacen sobre sí mismos. Esto último se vincula con la identidad y la generación de un sentido de pertenencia.

La representación que construyen los sujetos de su posición en el contexto social tiene un ingrediente más, el valor positivo o negativo (mejor o peor, inferior o superior), que le atribuyen al hecho de pertenecer a un grupo y no a otro. Esta situación de "valorización de sí mismo" respecto a los demás es lo que despierta en los sujetos el muy referido sentimiento de pertenencia, el orgullo de ser parte de ese grupo que goza de una imagen altamente valorada (Mercado, 2010, p. 239).

En el caso que se aborda, la representación que predomina acerca de los conductores del servicio que brindan las ERT es de ser superior a los conductores del servicio de transporte tradicional. La representación que hacen los conductores sobre sí mismos se ve fortalecida por la cultura de la organización que las mismas ERT se encargan de difundir entre los "socios" y ante la sociedad en general.

\section{INSTITUCIONES}

En el enfoque de la acción pública, por institución se entiende "los marcos de acción, es decir, reglas, rutinas, procedimientos que orienten las interacciones" (Lascoumes y Le Galés, 2014, p. 20). En dicho tenor, en este apartado se recupera parte de las estructuras normativas que regulan la prestación del servicio privado de transporte, debido a que la discusión generada sobre la ilegalidad de las ERT toma como base la normatividad existente en dicha materia.

Empero, es oportuno señalar que hablar de las instituciones implica también el referirse a las reglas del juego informales, que son compartidas y enmarcan las prácticas sociales que se dan en la cotidianidad. Cabe mencionar, que las instituciones formales no son neutras, es decir, que responden a intereses o lógicas que han logrado tener dominancia en contextos histórico-sociales específicos.

El marco regulatorio que rige la operación del servicio de transporte público en cDMx está conformado, en primera instancia, por la Constitución Política de los Estados Unidos Mexicanos, la Ley Federal de Competencia Económica, la Constitución Política de la Ciudad de México, la Ley de movilidad del Distrito Federal, el Reglamento de transporte del Distrito Federal, el Programa integral de movilidad para el periodo 2013-2018 y el Reglamento de la Ley de Movilidad del Distrito Federal. 
Una particularidad de la normatividad en esta entidad es que tanto la Ley de Transporte $y$ Vialidad del Distrito Federal (LtvdF), del año 2002, como la Ley de Movilidad (2014), que la abroga, contemplan al "Servicio Privado de Transporte", el cual es definido por la LTVDF como:

Es la actividad por virtud de la cual, mediante el permiso otorgado por la Secretaría, las personas físicas o morales satisfacen sus necesidades de transporte de pasajeros o de carga, relacionadas directamente ya sea con el cumplimiento de su objeto social o con la realización de actividades comerciales, sean éstas de carácter transitorio o permanente $y$ que no se ofrece al público en general (2002, p. 5).

En el artículo 12 de la LTvDF (2002), el servicio de transporte de pasajeros se clasifica en: público, mercantil, privado y particular. A su vez, el servicio privado comprende: escolar, de personal, turístico, especializado en todas sus modalidades y seguridad privada. Por su parte, en la Ley de Movilidad del Distrito Federal (2014), mantiene la misma definición de servicio privado de transporte, $y$ en su artículo 56 , refrenda la misma clasificación del servicio de transporte de pasajeros.

En esta modalidad se ampararon los portavoces de Uber y Cabify cuando se inició el conflicto, señalando que el servicio que prestan las ERT es un servicio privado y no público. Bajo esa consideración, las ERT defendían no estar compitiendo con los taxis ni tampoco incumpliendo con requisitos establecidos para el servicio público. Sin embargo, aún y cuando fuera de esa manera, sí incumplían en lo relativo a tener un permiso otorgado por la Secretaría.

En este caso, la regulación del servicio privado está amparada en el Código Civil del Distrito Federal (2015), específicamente en el Libro cuarto; Segunda parte; Título décimo, del contrato de prestación de servicios; Capítulo iv relativo a los porteadores $y$ alquiladores. A su vez, en el Código Fiscal vigente para el Distrito Federal, en su artículo 220, se establecen las cuotas que deberán pagar por el servicio de control vehicular diversos servicios de transporte, entre ellos, el servicio de transporte privado, aunque en dicho artículo solo se refiere al transporte privado escolar y de personal. Una particularidad es que el transporte de personal no conlleva un cobro, en tanto que traslada a individuos con una actividad en común. Estas lagunas legales fueron significativas, al permitirle a las ERT ganar tiempo e inducir una revisión y actualización de la normatividad.

Se podría decir que, si bien en la normatividad que aplicaba al Distrito Federal era permisible al servicio de transporte privado, el reclamo venía de los taxistas tradicionales, quienes consideraban una competencia desleal a las ERT, al no tener que cumplir con la regulación que se exige al transporte público en cuanto a pago de derechos (concesión, tarjetón), cromática, rótulos, medios de identificación oficiales, entre otros. Asimismo, al insistir que la modalidad de transporte que brindan las ERT cae dentro de la definición del servicio de transporte público en la modalidad individual. Inicialmente, las autoridades del gobierno capitalino respondieron a este reclamo, implementando operativos y reteniendo vehículos, y exigiendo el pago de una multa a los conductores; además, hicieron declaraciones públicas sobre la ilegalidad de las ERT.

\section{PROCESOS}

En el esquema de Lascoumes y Le Galés (2014) se menciona que la interacción entre actores, instituciones y representaciones da lugar a los procesos. Con fines expositivos, se ha dividido este apartado considerando tres momentos: la incursión de las ERT en CDMx, el conflicto generado y la regulación establecida.

\section{a) Incursión}

La entrada de las ERT, específicamente de Uber en la cDmx, siguió el mismo patrón que ha estado presente en todas las ciudades en las que anteriormente habían incursionado, comenzaron a circular vehículos prestando este servicio en el año 2013. En un principio no fue $\tan$ notoria la presencia de las ERT, ni para los prestadores del servicio de taxi tradicional, ni para la autoridad en materia de transporte. 
Probablemente, al inicio este significaba un asunto menor en comparación con el problema que representan las más de 50000 unidades de taxis piratas en la ciudad. El modus operandi de las ERT en las ciudades en las que han ido penetrando ha sido descrito de forma precisa:

1. Comienza operaciones, dejando de lado las disposiciones jurídicas vigentes, de forma ilegal o no regulada, en muchos de los casos, amparándose en el derecho privado (la contratación de un servicio entre particulares) ofreciendo ventajas competitivas como innovación $y$ mejor servicio. 2. Sus competidores (servicios de taxi y algunos servicios de transporte particular de pasajeros) buscan frenar su operación y solicitan la intervención de la autoridad responsable. 3. Se les prohíbe la operación, generan polémica y activan mecanismos de defensa orquestados por los propios usuarios del servicio. 4. Son regulados (Ávalos y Vásquez, 2015, p. 106).

Las ERT tuvieron una visibilidad suficiente y fueron interpeladas en la discusión pública, a la cual fueron yuxtaponiéndose otros actores, hasta lograr legalizar el servicio que brindan.

\section{b) Conflicto}

El crecimiento de usuarios de las ERT llevó a la confrontación física y legal con los prestadores del servicio de taxi, visibilizando las prácticas con las que se "solucionan" los problemas en el transporte público. La reacción de los usuarios no se hizo esperar. Estos actos, institucionalizados en el transporte público, incrementaron la reprobación social del servicio de transporte público.

Uno de los tantos hechos presentados en contra de Uber fue, en diciembre de 2014, cuando el líder de Taxistas Unidos Organizados del Distrito Federal presentó una denuncia ante la Procuraduría General de Justicia del Distrito Federal en contra del Secretario de Movilidad, por omisión; a los representantes de las ERT, y los taxis piratas que circulan en la ciudad. Esta denuncia evidenció no solo la pugna de los taxis tradicionales con el servicio de transporte bajo demanda, sino también la falta de atención en la regulación del transporte público (El Economista, 10/12/2014).

De forma intermitente comenzaron enfrentamientos $y$ contraataques entre taxistas del Distrito Federal y operadores de las ERT, sin que mediara una solución. El 25 de mayo de 2015 , se llevó a cabo una manifestación de taxistas, en la que se bloquearon diversos puntos estratégicos de la ciudad, acto que fue cubierto por las televisoras del país. Como resultado, las autoridades capitalinas acordaron incrementar los operativos contra taxis piratas. Las constantes pugnas $y$ bloqueos que siguieron por la presencia de Uber trajeron consigo algunos exhortos de la Asamblea Legislativa del Distrito Federal para llevar a cabo mesas de diálogo entre los interesados.

El 4 de junio de 2015, la cofece emitió una recomendación dirigida a los Gobernadores de los Estados, el jefe de gobierno del Distrito Federal $y$ las Legislaturas de las entidades federativas. En dicha recomendación, la $\operatorname{cofEcE}^{8}$ resaltó el impacto favorable del servicio de transporte que ofrecen las ERT para los consumidores e instó a las autoridades gubernamentales a reconocer esta modalidad de transporte como una categoría diferente a la del servicio de taxi. La COFECE insistió en evitar restricciones como:

a) Autorizar o registrar vehículos para prestar el servicio o limitar su número imponiendo requisitos adicionales como de placas especiales $y / o$ cromáticas; $y$

b) Regular los esquemas tarifarios, los cuales actualmente son determinados por las propias ERT en función de la oferta y demanda del mercado (COFECE, 2015, p. 7).

8 Con esta recomendación, la COFECE participó en el Concurso de promoción de la Competencia 20152016, que organizan el Banco Mundial y la Red Internacional de Competencia, resultando ganadora en la categoría de promoción a la competencia en los mercados innovadores. 
En ese mismo mes se llevó a cabo el Debate Digital en la cDmx, en el marco de la Ley de Ciudad Abierta, en cuyas disposiciones establece el derecho de los particulares a participar colaborativamente en la conformación, desarrollo y evaluación de programas, políticas y acciones de gobierno. Este tipo de procesos resulta consustancial a la construcción de legitimidad de las decisiones públicas en un contexto de democracia participativa.

Sin embargo, en las discusiones por medio de redes sociales, el mejor manejo de estas redes, ya sea por capital cultural o mercadológico, influyó en el peso de cada uno de los actores intervinientes. La activa participación de la ciudadanía en el Debate Digital, en apoyo a que Uber $y$ Cabify se quedaran en el mercado de la cDmx, constituye una particularidad de esta ciudad, distinto a lo ocurrido en otras entidades del país.

Por su parte, la Comisión de Derechos Humanos del Distrito Federal manifestó que la actividad de las ERT constituye una modalidad diferente a la que ofrece el servicio público de transporte concesionado, conocido como taxi, exhortando al Gobierno de la Ciudad a revisar la normatividad que se les aplica a estos últimos, a fin de evitar sobre regulaciones. Asimismo, instó a permitir elegir libremente la forma de traslado, lo cual, señaló, se encuentra previsto en la Ley de Movilidad (CDHDF, 2015).

El 2 de julio de 2015, los periódicos de circulación nacional anunciaron que el Gobierno del Distrito Federal condonaría multas a concesionarios que no cumplieron con la revista ${ }^{9} 2015$ y daría un bono de chatarrización, junto a la promesa de generar un "piso parejo"

$9 \quad$ De acuerdo con la Ley de transporte $y$ vialidad del Distrito Federal, toda unidad de transporte concesionado debe contar con la revista vehicular, definida como la inspección física de las unidades, equipamiento auxiliar o infraestructura de los servicios de transporte público y privado, a fin de comprobar el cumplimiento de las disposiciones en materia de instalaciones, equipo, aditamentos, sistemas y en general, las condiciones de operación y especificaciones técnicas para la óptima prestación del servicio. Por la revista se debe realizar un pago anual, y el automóvil que no cuente con ella puede ser remitido a corralón. ante la inminente regulación de las ERT en la cDmx. Con estas medidas, el gobierno local estabiliza momentáneamente las movilizaciones.

\section{c) Regulación}

Con fecha 15 de julio de 2015, el Gobierno del Distrito Federal, a través de la Secretaría de Movilidad, publicó en su Gaceta Oficial número 133bis, el Acuerdo por el que se crea el registro de personas morales que operen y/o administren aplicaciones y plataformas informáticas para el control, programación y/o geolocalización en dispositivos fijos o móviles, a través de las cuales los particulares pueden contratar el servicio privado de transporte con chofer en el Distrito Federal. También, en esa misma fecha, se crea el Fondo para el Taxi, la Movilidad y el Peatón, el cual establece que se debe de integrar por el equivalente al 1,5\% de los ingresos por viajes realizados por los prestadores de servicio privado de transporte a través de aplicaciones.

A su vez, la Ley de Movilidad señala que los prestadores de servicios de transporte privado de pasajeros deberán contar con un permiso expedido por la Secretaría de Movilidad $y$ registrar ante esta, las unidades integradas en sus plataformas (el registro tendrá una vigencia anual), así como a los conductores de los vehículos. Posterior al Acuerdo de fecha 15 de julio de 2015, se han dictado otro Acuerdo y Lineamientos en los que se adicionan o modifican disposiciones establecidas, como lo son los requisitos para la obtención de la constancia de registro vehicular ${ }^{10}$.

$10 \quad$ El 12 de agosto de 2016, se publica en la Gaceta Oficial de la Ciudad de México, un Acuerdo en el que se adicionan y modifican algunos numerales del Acuerdo de fecha 15 de julio de 2015. Disponible en el siguiente link: http://data.consejeria.cdmx.gob. mx/portal_old/uploads/gacetas/0c37b1746512f388 bf98ff67e80bcd33.pdf

Posteriormente, el 6 de septiembre de 2016 , se publica en la Gaceta Oficial de la Ciudad de México, la convocatoria para la prestación del servicio de "validación vehicular anual", para el cumplimiento al numeral noveno del Acuerdo publicado en la Gaceta Oficial de la Ciudad de México, con fecha 12 de agosto de 2016, que modifica el diverso 
Será hasta el 15 de septiembre de 2017, que se publique el Reglamento de la Ley de Movilidad del Distrito Federal, cuando se establece la siguiente definición:

Servicio Privado de Transporte con Chofer: Servicio de transporte que pueden contratar los particulares a través de las personas morales que operen $y / 0$ administren aplicaciones $y$ plataformas informáticas para el control, programación y/o geolocalización en dispositivos fijos o móviles que cuenten con el permiso correspondiente otorgado por la Secretaría (RLMdF, 2017, p. 3).

En la sección octava de este Reglamento, artículos 57, 58 y 59, se establecerá la regulación para esta modalidad de transporte de pasajeros, destacando que:

El Titular de la Constancia de Registro o Plataforma Móvil es el obligado a realizar el pago de derechos por cada vehículo aprobado y registrado que estipula el artículo 220 fracción I, inciso d, numeral 1, 1.2 del Código Fiscal de la Ciudad de México, así como a verificar el cumplimiento de la Validación Vehicular Anual en los términos y mecanismos que determine la Secretaría para los efectos (RLMDF, 2017, p. 17).

\section{RESULTADOS}

Luego de un relativamente rápido proceso de confrontación y negociación se culminó con la regulación de las ert en la cDMx. A más de dos

por el que se crea el registro de personas morales que operen $y / 0$ administren aplicaciones $y$ plataformas informáticas para el control, programación y/o geolocalización en dispositivos fijos o móviles, a través de las cuales los particulares pueden contratar el servicio privado de transporte con chofer en el distrito federal. Los requisitos se pueden consultar en la siguiente liga:

http://data.consejeria.cdmx.gob.mx/portal_old/ uploads/gacetas/629955ef26f1e407e66407c44a273 7b7.pdf años de puesto en vigor el Acuerdo del 15 de junio de 2015, se presentan vicios de antaño. En primer lugar, la falta de control por parte de las autoridades gubernamentales en materia de transporte sigue siendo una tarea pendiente. Ello ha sido evidenciado por la opacidad en la que se mantiene el manejo de los recursos provenientes del Fondo para el Taxi, la Movilidad y el Peatón.

Distintos actores sociales han hecho uso del derecho a solicitar información por medio del Instituto de Transparencia, para que la sEмоvi informe sobre los recursos obtenidos del Fondo en mención. Lo anterior, aunado a la coexistencia del poder federal y local en la cDmx, provenientes de partidos políticos con ideologías diferentes, y el pluralismo político en los órganos legislativos, han favorecido un debate continuo sobre el funcionamiento de las ERT. Por ejemplo, un diputado del grupo parlamentario del Partido Acción Nacional (pan) solicitó, el 17 de febrero de 2016, ante la Asamblea Legislativa del Distrito Federal, que se entregue un informe de ingresos y egresos del Fondo; sin embargo, la información no se ha otorgado. A su vez, el 23 de febrero de 2017, el Grupo Parlamentario del Partido Revolucionario Institucional (PRI) del Senado de la República presentó un exhorto al Gobierno de la Ciudad de México comx para que transparente el monto de los recursos aportados este Fondo, así como, las obras o acciones específicas en las que se han aplicado dichos recursos.

Adicionalmente, la credibilidad en el servicio de las ERT ha sufrido algunas mermas debido a ciertas acciones, como lo fue la respuesta que dieron a los usuarios de este servicio ante la contingencia ambiental decretada el 6 de abril de 2016 en la comx, a partir de la que el $40 \%$ del parque vehicular dejó de circular, favoreciendo un incremento en la demanda de viajes, lo que propició que las tarifas de las ERT, en específico de Uber, aumentaran de forma significativa en ciertas horas pico.

Así, la tarifa dinámica, es decir, el pago de acuerdo con las condiciones de oferta y demanda en tiempo real, tuvo un impacto negativo para los usuarios, lo que desató una fuerte crítica hacia el servicio de esta ERT. De nueva cuenta, las redes sociales sirvieron para presionar ante este 
hecho, de tal suerte que el gobierno de la capital entabló comunicación con la empresa Uber, quién puso restricciones a la tarifa dinámica.

Por otro lado, la indisciplina de los operadores de las ERT no se ha hecho esperar. Para ilustrar lo anterior, en las restricciones de este servicio se determinó que los vehículos de transporte individual de pasajeros con aplicaciones móviles no tienen permitido hacer base o establecer un sitio. El Instituto de Verificación Administrativa (Invea) y la Secretaria de Seguridad Pública del Distrito Federal son los encargados de inspeccionar y verificar que se cumpla con lo establecido. El Invea ha sancionado a los conductores de las ERT por incumplimiento y los diarios de circulación nacional también han mostrado dicha realidad.

Otro territorio en disputa lo es el aeropuerto de la cDmx. La normatividad señala que el servicio de transporte dentro del aeropuerto tiene que suscribir un contrato de acceso a la zona federal y pagar una contraprestación. Los conductores de Uber han replicado una vez más su estrategia de incumplir la norma para provocar que los reflectores de los medios y los usuarios del servicio se enfoquen en dicha reglamentación. Sin embargo, en octubre de 2017, los prestadores del servicio de transporte de pasajeros del Aeropuerto Internacional de la Ciudad de México ganaron un amparo, que obliga a las autoridades federales en la materia a impedir el transporte de personas desde el aeropuerto si no se cuenta con el permiso o concesión federal (Diario Visión de Michoacán, 2017).

Paso a paso, las eRT buscan modificar las restricciones establecidas en la regulación que se dio en la cDmx. En dicha entidad, la normatividad para este servicio especifica que no se permite recibir pagos en efectivo. Esta característica del servicio que prestan las ERT fue uno de los principales argumentos que utilizó la cofece para decir que no existía competencia con los transportistas del servicio público, debido a que cada uno atiende a segmentos específicos del mercado, que en el caso de las ERT son usuarios que tienen celular con acceso a internet y cuentan con servicios bancarios para hacer pago con tarjeta.

Sin embargo, en otros Estados de la República, como el Estado de México, la regulación establecida posterior a la de la cDMx, permitió el pago en efectivo. En este orden de ideas, el Director de Uber en la cDmx, Roberto Fernández del Castillo, señaló públicamente que se puede mejorar el servicio en el rubro de cobros en efectivo y validación de los vehículos, pero ello es una decisión del gobierno (López, 2012).

En Yucatán, derivado de la acción de inconstitucionalidad $63 / 2016$ promovida por diputados de la legislatura de dicha entidad federativa, se demandó la invalidez de diversas disposiciones de la Ley de Transporte local, en materia de transporte de pasajeros contratado mediante aplicaciones (apps). Como resultado, volvió a entrar en discusión qué tipo de servicio prestan las ERT; determinando que no prestan el servicio de transporte, $y$ que son empresas de naturaleza mediadora entre el usuario y el operador. Las diversas interpretaciones acerca del tema se observan cuando una ministra votó en contra de si el Congreso del estado de Yucatán tiene o no competencia para regular en materia de transporte, toda vez que manifestó la duda acerca de si las ERT están realizando actos de comercio, si estos se tipifican como actos de comercio electrónico, y en este segundo supuesto, si es competencia de las entidades federativas (scss, 2017).

En otro orden de ideas, un factor que ha abonado a la pérdida de confiabilidad en la seguridad de las ERT han sido los acontecimientos delictivos en los que han estado involucradas unidades y operadores de las ERT Uber y Cabify, evidenciando la falta de control de la empresa sobre los operadores del servicio y la reducción en los requisitos de sus procesos de reclutamiento. Por ejemplo, al principio Uber en el proceso de selección de los "socios" de Uber, el candidato tenía que presentar una prueba de ubicación por la ciudad, de servicios, examen de manejo, antidopaje, examen psicométrico, carta de antecedentes no penales, registro ante el Sistema de Administración Tributaria (sat), además de otros filtros. En la actualidad, Uber realiza el proceso de "selección" en línea. Pese a todo, el fenómeno de las ERT sigue en expansión. En esa misma tesitura, en diciembre de 2017, Uber realizó un cambió en sus términos y condiciones, en los que establece que la empresa no se hace 
responsable de cualquier incidente que ocurra durante el viaje.

\section{CONCLUSIONES}

En este artículo se ha buscado establecer la configuración de la acción pública ante la entrada de las ERT en CDMx, una nueva modalidad en la prestación del servicio de transporte individual de pasajeros, cuya presencia ha venido a trastocar el modelo de gestión del transporte público de pasajeros.

Se ha utilizado el enfoque de la acción pública al considerarse que, a través de esta, es posible dar cuenta de distintas dimensiones que entraron en juego en la regulación de las ERT en la CDMx, esto es, actores con intereses distintos, instituciones que se reproducen $y$ otras más que se recomponen, representaciones e identidades que favorecen la acción colectiva. A partir de lo anterior, el arreglo establecido, que permite la coexistencia tanto del servicio público como del privado, es entendido como un sistema de orden negociado. Esto es distinto a las posturas reduccionistas que abordan las decisiones públicas de manera vertical y sin contradicciones, desde el proceso de elaboración y hasta su implementación. En este enfoque de la acción pública es necesario situarse en los espacios en los que se hacen y rehacen las clientelas $y$ coaliciones de grupos de interés.

De esta manera, se pudo dar cuenta de la particular configuración de la relación gobierno-ciudadanía que se da en la cDMx, en donde esta última ejerce su capacidad de agencia en los asuntos públicos y utiliza los recursos tecnológicos para opinar sobre aquellos temas que requieren de la intervención de las autoridades gubernamentales. En esta nueva relación, también se recompone la función del gobierno, quién debe construir consensos, toda vez que la legitimidad es un asunto político y no un aspecto técnico.

En el desarrollo de este documento se presentaron distintos momentos del proceso de estructuración de un nuevo orden, el cual se va tejiendo mediante intereses contradictorios $y$ discrepancias con el ordenamiento existente, negociaciones políticas, participación ciudadana intermitente; en la cual entran en juego elementos objetivos y subjetivos. Dentro de los elementos objetivos se encuentran instituciones condicionantes y que han sido resultado de procesos histórico-sociales particulares, ante los cuales fueron una respuesta. En la parte subjetiva, están las representaciones sociales sobre aspectos vinculados a los dos tipos de servicios de transporte, de los que se ha hecho mención en este artículo, pero también la representación que los principales actores se van haciendo sobre sí mismos.

El resultado de la interacción y confrontación social que se dio ante la incursión de las ERT en la CDMx son nuevas elaboraciones institucionales, las que luego se constituyen en la condición de las interacciones entre los actores. Esto sin dejar de lado que los arreglos se harán dependiendo de las relaciones de poder y correlación de fuerzas que se presente en el contexto de acción, institucionalizándose la postura que tenga mayor fuerza. En este caso, legitimadas por la sociedad de forma previa.

Nuevos aspectos se muestran e invitan a la reflexión y análisis, tal es el caso de la reconfiguración de la fragmentada identidad de los operadores del servicio de transporte público ante la presencia de un nuevo "otro". La capacidad de movilización y organización de los concesionarios de transporte público se ha dado desde su idiosincrasia, pero también han adoptado nuevos mecanismos que les han permitido conformar frentes nacionales e internacionales, mediante alianzas temporales, fortaleciendo la identidad de un gremio con una representación deteriorada.

Como colofón, las ERT lograron legitimarse en la cDmx bajo el argumento de la mejora en la calidad del servicio de transporte. Así, luego de la legitimación en la sociedad, impulsada por las тics, vino la legalización, mediante el establecimiento de una nueva normatividad en la que las ERT han sido incluidas. Diversos actores, intereses $y$ representaciones apuntaron a encauzar la parte legal a favor de las ERT. Sin embargo, a la fecha sigue habiendo posturas que señalan que no era necesaria la regulación $y$ hay quienes insisten en la necesidad de establecer límites. 
Se tiene un orden legal, empero lo social se caracteriza por la complejidad y por ser contradictorio. La legitimidad, aún y cuando tenga un sustento legal, ya no es estable, requiere de una demostración permanente de la efectividad, $y$ ese es el gran reto de las ERT. Ello se puede constatar con el cobro de tarifas adicionales que propone Uber, si el conductor espera más de dos minutos, por un lado, y la campaña llamada "7 acciones", por otro. Uber no solo tiene que ponerse del lado del usuario, sino también del lado del conductor.

Adicionalmente, la reciente resolución del Tribunal de Justicia de la Unión Europea, emitida el 20 de diciembre de 2017, dicta que Uber es una empresa de transporte y no solamente una aplicación digital que contacta choferes con usuarios.

...y, por lo tanto, ha de calificarse de "servicio en el ámbito de los transportes", a efectos del Derecho de la Unión. En consecuencia, un servicio de esta índole está excluido del ámbito de aplicación de la libre prestación de servicios en general, así como del ámbito de aplicación de la Directiva relativa a los servicios en el mercado interior y del de la Directiva del comercio electrónico (тנUE, 2017, p. 1).

Con esta sentencia, podrá reaparecer la discusión sobre la regulación a las ERT.

\section{REFERENCIAS}

Abascal, A. (2009). El servicio público de taxi. En Fernández Ruíz, Jorge; Germán Cisneros Farías y Filiberto Otelo Salas (coord.). Régimen Jurídico del Urbanismo. Memoria del Primer Congreso de Derecho Administrativo Mexicana, México, unam, pp. 225-257.

Acuerdo por el que se crea el Registro de Personas Morales que Operen y/o Administren Aplicaciones y Plataformas Informáticas para el Control, Programación y/o Geolocalización en Dispositivos Fijos o Móviles, a través de las cuales los Particulares pueden Contratar el Servicio Privado de Transporte con Chofer en el Distrito Federal. Recuperado de http://www.transparencia. df.gob.mx/work/sites/vut/resources/ ExcelContent/17368/1/15072015.pdf

Ávalos, M. y Vásquez, P. S. (enero-febrero de 2015). Baby, you can ('t) drive my car. El caso de Uber en México. Economía informa, 390, 104-112. Recuperado de http://www.economia.unam.mx/assets/ pdfs/econinfo/390/06avalosysofia.pdf

Código Civil para el Distrito Federal. (2015). Última reforma publicada el 05 de febrero de 2015. Recuperado de http://www. aldf.gob.mx/archivo-c9dc6843e50163a0d2628615e069b140.pdf

Código Fiscal del Distrito Federal. (2012). Reforma publicada el 31 de diciembre de 2012. Recuperado de https://data.finanzas.cdmx.gob.mx/transparencia/docs/ art14/fraccI/cod_fisc_df.pdf

cofece. (04/06/2015). Pleno de la Comisión Federal de Competencia Económica. Opinión OPN-008-2015. Recuperado de http://www.cofece.mx:8080/cfcresoluciones/docs/Mercados\%20Regulados/ V6/16/2042252.pdf

Comisión de Derechos Humanos del Distrito Federal. (2015). cDHDF observa diálogo de gobierno con concesionarios de taxis y empresas como Uber y Cabify. Dirección General de Comunicación por los Derechos Humanos. Boletín de prensa 142/2015, México. Recuperado de http://cdhdf.org.mx/wp-content/ uploads/2015/06/Bol1422015.pdf

Conflicto de taxistas contra Uber y Cabify llega a la pgJdf. (10/12/2014). El Economista. Recuperado de http://eleconomista.com. $\mathrm{mx} /$ tecnociencia/2014/12/10/conflictotaxistas-contra-uber-cabify-llega-pdf

Constitución Política de la Ciudad de México. (5 de febrero de 2017). Gaceta Oficial de la Ciudad de México, México.

De la Garza, E. (mayo-agosto 2013). Trabajo no clásico y flexibilidad. Cuaderno CRH, 26 (68), 315-330, Recuperado de http://www. scielo.br/scielo.php?script=sci_arttext\&pi $\mathrm{d}=$ S0103-49792013000200007 
Ganan amparo taxistas del Aeropuerto de la cDmx; podrán replicarlo en estados. (12/10/2017). Diario Visión de Michoacán. Recuperado de http:// www.eldiariovision.com.mx/noticia/ nota,61517/t,Ganan+amparo+taxistas+de $1+$ Aeropuerto $+\mathrm{de}+\mathrm{la}+\mathrm{CDMX} \% 3 \mathrm{~B}+$ podr $\% \mathrm{C}$ $3 \% \mathrm{~A} 1 \mathrm{n}+$ replicarlo+en+estados+/

Gobierno del Distrito Federal. (30/12/2003). Reglamento de Transporte del Distrito Federal. Gaceta Oficial del Distrito Federal, Décima tercer época, №. 102TER. Recuperado de http://data.consejeria. cdmx.gob.mx/portal_old/uploads/gacetas/ diciembre03_30_102_ter_I.pdf

González de Cossío, F. (s/f). El caso Uber en México. Un Cri de Coeur. Recuperado de http:// www.gdca.com.mx/PDF/competencia/ EL\%20CASO\%20UBER.pdfEGII

Hernández, Y. y Galindo, R. V. (septiembrediciembre de 2016). Modelo de gestión del servicio de transporte Uber. ¿Quién pierde y quién gana? Revista Espacios Públicos, 19 (47), 157-175. Recuperado de http://www.redalyc.org/ pdf/676/67650281008.pdf

INFomEx. (2017). Respuesta a la solicitud de información pública 0106500018517 otorgada por la Dirección operativa de la Dirección general del servicio de transporte público individual y enlace con la unidad de transparencia de la secretaria de movilidad de la Ciudad de México, el día 13 de febrero de 2017.

Instituto Nacional de Estadística y GeografíaINEGi. (2017). Encuesta Origen-Destino en Hogares de la Zona Metropolitana del Valle de México, México.

Instituto Nacional de Estadística $y$ Geografía (INegi)-Universidad Nacional Autónoma de México (unam). (2017). Estudio Origen-Destino de la Zona Metropolitana del Valle de México 2017. [Diapositivas] Recuperado de http://www.iingen.unam.mx/es-mx/ Investigacion/Proyectos/Documents/ PresentacionOD.pdf

Jiménez Bautista, F. (2009). Concesión del transporte público individual. Actualidad de los servicios públicos en México. En D. Cienfuegos y L. G. Rodríguez (coord.) (pp. 217-247). México: Biblioteca jurídica virtual del Instituto de Investigaciones Jurídicas de la Universidad Nacional Autónoma de México (unam). Recuperado de https://archivos.juridicas.unam.mx/ www/bjv/libros/6/2654/2.pdf

Laboratorio para la Ciudad-(2015). Debate digital cDmx. Realizado los días 17 y 18 de junio de 2015. Recuperado de: http:// labcd.mx/debate-digital-cdmx/

Lascoumes, P. y Le Galés, P. (2014). Sociología de la acción pública. Vicente Ugalde (traductor). México: El Colegio de México.

Law@Tec. (24/06/2016). La Batalla de Uber en México. Escuela de Gobierno y Transformación Pública, Tecnológico de Monterrey. Recuperado de https://posgrado derecho.itesm.mx/blog/Uber-en-Mexico/

Ley de Movilidad del Distrito Federal. (14/07/2014). Asamblea Legislativa del Distrito Federal, vi Legislatura. Recuperado de http://www.aldf.gob.mx/ archivo-ba20960fb6570ec7d4ee34c30e e2d733.pdf

Ley de Transporte y Vialidad del Distrito FederalLtvdF. (2002). Asamblea Legislativa del Distrito Federal iv Legislatura. Recuperado de http://docs.mexico. justia.com.s3.amazonaws.com/estatales/distrito-federal/ley-de-transporte$y$-vialidad-del-distrito-federal.pdf

López, J. (08/12/2016). Uber planea cobrar en efectivo en la Ciudad de México. Excelsior.com.mx. Recuperado de http:// www.excelsior.com.mx/comunidad/ 2016/12/08/1133081

Medina, J. (20/12/2013). El origen de los taxis en México. Recuperado de http://javiermedina loera.com/arrierosdemexico/?p=198

Mercado, A. y Hernández, A. V. (mayo-agosto de 2010). El proceso de construcción de la identidad colectiva. Revista Convergencia, 52, 229-251. Recuperado de http://www.scielo.org.mx/pdf/conver/ v17n53/v17n53a10.pdf 
Mora, M. (2002). La teoría de las representaciones sociales de Serge Moscovici. Athenea Digital, 2. Recuperado de http://www. raco.cat/index.php/Athenea/article/view File/34106/33945

Moreno, S. S; López, S; García, M. y Ruíz, J. A. (2016). Impacto del servicio del modelo de chofer particular apoyado en tecnología. Caso Uber. En Innovando a través de Sotfware. Mónica García Munguía, et al (editores). Editorial Academia Dragón Azteca S. de R.L. de c.v., Cuernavaca Morelos. Recuperado de https://www. researchgate.net/publication/313746638_ Capitulo_XII_Impacto_del_servicio_del_ modelo_de_chofer_particular_apoyado_ en_tecnologia_Caso_Uber

Parra, F. J. (mayo-junio de 2017). Uber y la tortura institucionalizada en Yucatán. Revista del IIJ, Hechos y Derechos, 29. Recuperado de https://revistas.juridicas. unam.mx/index.php/hechos-y-derechos/ article/view/11345/13279

Pogliaghi, L. (2012). Entre el control y la libertad: configuraciones de trabajo, identidad y acción colectiva de los taxistas de la Ciudad de México. (Tesis de doctorado en Estudios Sociales), Universidad Autónoma Metropolitana, Unidad Iztapalapa, México.

Ramos, L. A. (abril de 2011). Los servicios públicos y la concesión administrativa. Lecturas Jurídicas, 15. Recuperado de http://fd.uach.mx/maestros/2011/06/20/ Revista_Lecturas_Juridicas15.pdf
Reglamento de la Ley de Movilidad del Distrito Federal. (2017). Gaceta Oficial de la Ciudad de México. Recuperado de http:// data.consejeria.cdmx.gob.mx/portal_old/ uploads/gacetas/585b39baccbd5bc797ba 4e3ffe84d060.pdf

Suprema Corte de Justicia de la Nación. (scun, 2017). Sesión Pública nro. 44 Ordinaria, 22/05/2017. Recuperado de https:// www.scjn.gob.mx/sites/default/files/ actas-sesiones-publicas/documento/ 2017-05-26/44_0.pdf

Thoening, J-C. (1997). Política pública y acción pública. Gestión y Politica Pública, vi (1), 19-37. Recuperado de http://www.aleph.org.mx/jspui/bitstream/56789/11713/1/DOCT2064257_ ARTICULO_2-VOLVINUM1.PDF

Tribunal de Justicia de la Unión Europea-tue. (20/12/2017). Comunicado de Prensa, núm. 136/17. El servicio de puesta en contacto con conductores no profesionales prestados por Uber está comprendido en los servicios en el ámbito de los transportes. Luxemburgo.

Vergara, J. (julio-diciembre de 2005). La concepción de la democracia deliberativa de Habermas. Quórum Académico, 2 (2), 72-88. Recuperado de http://www. redalyc.org/articulo.oa?id=199016762004

Fecha de ingreso: 10/10/2017 Fecha de aprobación: 25/06/2018 
